\title{
Diseño y automatización de celda de dispensado y pegado de material cerámico mediante lámparas UV
}

\section{Design and automation of ceramic material dispensing and gluing cell using UV lamps}

MAYORQUÍN-ROBLES, Jesús Antonio†*, MEDINA-MUÑOZ, Luis Arturo, MELÉNDEZ, Gisela Guadalupe y RODRÍGUEZ-SENDAY, José Jesús

TECNM/Instituto Tecnológico de Nogales. Departamento de Metal Mecánica

ID $1^{\text {er }}$ Autor: Jesús Antonio, Mayorquín-Robles / ORC ID: 0000-0002-3438-2098, Researcher ID Thomson: H-40712018, CVU CONACYT ID: 471236

ID $1^{\text {er }}$ Coautor: Luis Arturo, Medina-Muñoz / ORC ID: 0000-0001-9598-1451, Researcher ID Thomson: H-4735-2018, CVU CONACYT ID: 454558

ID 2 ${ }^{\text {do }}$ Coautor: Gisela Guadalupe, Meléndez / ORC ID: 0000-0003-1391-4170, CVU CONACYT ID: 903761

ID $3^{\text {er }}$ Coautor: José Jesús, Rodríguez-Senday / ORC ID: 0000-0001-8457-0020, I-2702-2018, CVU CONACYT ID: 904262

DOI: $10.35429 / J E A .2020 .22 .7 .8 .13$

Recibido: Enero 15, 2020; Aceptado: Marzo 28, 2020

\begin{abstract}
Resumen
La implementación de las nuevas tecnologías dentro de las áreas de diseño y automatización, desempeñan un puesto relevante en los procesos de fabricación, sobre todo cuando se busca aumentar la eficiencia y volumen dentro de las líneas de producción, mejorando la calidad de los productos, reduciendo desperdicios, aumentando la seguridad, así como crear espacios ergonómicos de trabajo. El presente trabajo se realizó con la finalidad de mejorar los niveles de calidad y productividad en el proceso de dispensado y pegado en el ensamble de un sensor, debido a las demandas de los clientes, se decidió diseñar y automatizar una celda para llevar a cabo esta operación con mayor eficiencia. Mediante el uso de herramientas de diseño mecánico y software, como Solid Works, el cual ayudó a establecer el diseño de la celda de manufactura, PLC'S, Driver's y Servomotores, los cuales en conjunto logran la integración de ambas ramas.
\end{abstract}

Automatización, Implementación, Tecnologías, Eficiencia

\begin{abstract}
The implementation of new technologies within the areas of design and automation, play a relevant role in manufacturing processes, especially when seeking to increase efficiency and volume within production lines, improving the quality of products, reducing waste, increasing safety, as well as creating ergonomic work spaces. The present work was carried out with the purpose of improving the levels of quality and productivity in the process of dispensing and gluing in the assembly of a sensor, due to the demands of the clients, it was decided to design and automate a cell to carry out this operation with greater efficiency. Through the use of mechanical design tools and software, such as Solid Works, which helped establish the design of the manufacturing cell, PLC'S, Driver's and Servomotors, which together achieve the integration of both branches.
\end{abstract}

Automation, Implementation, Technologies, Eficiency

Citación: MAYORQUÍN-ROBLES, Jesús Antonio, MEDINA-MUÑOZ, Luis Arturo, MELÉNDEZ, Gisela Guadalupe y RODRÍGUEZ-SENDAY, José Jesús. Diseño y automatización de celda de dispensado y pegado de material cerámico mediante lámparas UV. Revista de Aplicaciones de la Ingeniería. 2020. 7-22: 8-13.

\footnotetext{
$\dagger$ Investigador contribuyendo como primer autor.
} 


\section{Introducción}

El término automatización se propuso en los años cuarenta en Estados Unidos para indicar el manejo y proceso automáticos de las máquinas en la producción. Actualmente, se considera que la automatización no es una opción para mantenerse en el mercado, sino una necesidad. Los sistemas de manufactura existentes se han transformado gracias al desarrollo de las máquinas/herramientas, la implementación de software, herramientas de diseño y control de la producción, entre otros [1].

La competencia en el sector industrial y los altos estándares que demandan los clientes exigen al mercado la mejora continua de sus procesos. La industria abunda en oportunidades para la implementación racional de automatismos y la ingeniería juega un papel importante para identificar dichas oportunidades y de esa manera alcanzar y superar dichas demandas $[2,3]$.

El formular un plan para satisfacer una necesidad especifica o resolver un problema se conoce como proceso de diseño. Es por ello que el proceso de diseño es innovador, altamente iterativo y requiere de una toma acertada de decisiones.

En la actualidad, existe una gran variedad de herramientas y recursos disponibles que ayudan en la solución de problemas de diseño. El software para el diseño asistido por computadora (CAD) permite el desarrollo de diseños tridimensionales a partir de los cuales pueden producirse vistas ortográficas convencionales en dos dimensiones con dimensionamiento automático. Otra ventaja de este tipo de base de datos es que permite cálculos rápidos y exactos de ciertas propiedades como la masa, la localización del centro de gravedad y los momentos de inercia de masa. Del mismo modo, pueden obtenerse con facilidad otras propiedades como áreas y distancias entre puntos. Existe una gran cantidad de software de CAD disponible como Aries, AutoCAD, CadKey, I-Deas, Unigraphics, Solid Works y ProEngineer, entre otros [4].
En la producción de sensores utilizados en sonares militares (Del inglés SONAR, acrónimo de Sound Navigation And Ranging, que significa "navegación por sonido") se ha presentado la problemática de que el sensor al momento de ensamblarlo en el tubo base no queda del todo concéntrico, provocando así una deficiencia en su función. El siguiente equipo fue diseñado para llevar a cabo una operación de dispensado y pegado por medio de lámparas UV de un material cerámico, en el cual se mantiene constante la cantidad de adhesivo y así el problema de la concentricidad del cap y el tubo se erradica.

\section{Diseño y Automatización del Sistema}

Después de un análisis inicial del proceso de dispensado y pegado de un material cerámico que se realizaba de manera manual para un sonar militar, se procedió a buscar una solución para arreglar la problemática de la falta de concentricidad que existía entre el cap y su tubo base. Para ello, se propuso el crear dos estaciones de trabajo, una donde se hará el proceso de dispensado y la segunda, donde se realizará el proceso de curado. Al definir esto, se hizo búsqueda de elementos que servirían para satisfacer los requerimientos de los procesos.

En el diseño del sistema, se decidió instalar un dispensador de la marca Loctite (ver Fig. 1), que combina el controlador dual de dispensado con un depósito en un solo sistema [5]. Este dispensador cuenta con dos puertos de activación electrónica, donde cada uno va a ser para una estación de trabajo. Gracias a su cámara de presurización neumática, se puede llevar el adhesivo hasta las dos válvulas reguladoras de flujo cuyo trabajo es el mantener constante el adhesivo que se le aplica a cada pieza a través de jeringas dispensadoras de alta precisión [6].

Para agilizar el dispensado de cada pieza, se buscó diseñar un fixture de acuerdo a las especificaciones de la pieza. Este fixture está acoplado a un servomotor para hacer más precisa la llegada a cada notch del tubo. Para la alineación de la aguja y el notch, se instaló un sensor fotoeléctrico de la marca Keyence, con una distancia focal de 10 a $30 \mathrm{~mm}$ y resistencia de temperatura de $-40 \mathrm{a}+70{ }^{\circ} \mathrm{C}$, para detectar la parte donde se encuentra el notch [7]. 


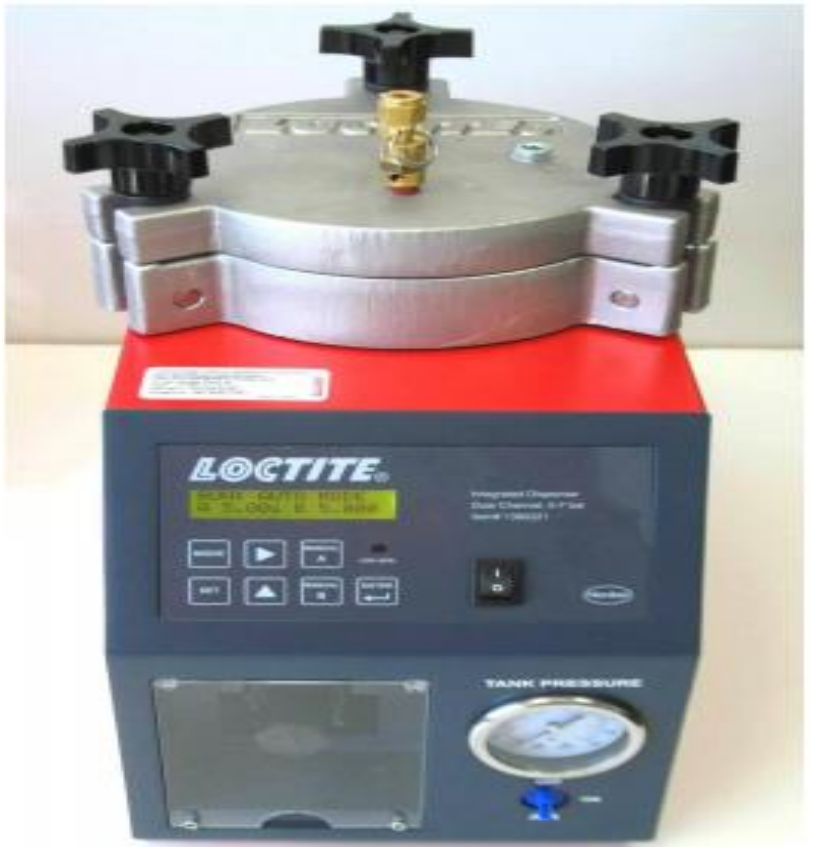

Figura 1 Dispensador de Loctite

En el área de curado de adhesivo, se diseñó una prensa neumática. Esta prensa está montada a un servomotor el cual la hace girar para que la pieza reciba la luz ultra violeta uniformemente por toda su circunferencia.

La estación 2 a diferencia de la estación 1 tiene una prensa extra de curado, ya que la estación 2 lleva mayor tiempo de curado y requiere una extra para balancear la operación. Para el manejo y visualización de la operación, se implementó una pantalla táctil HGIF de la interfaz de la familia de IDEC SmartTouch (ver Fig. 2). Las ventajas de este panel es que cuenta con las características de un panel de tamaño normal en una versión más pequeña, siendo adaptable a espacios reducidos y con la versatilidad de implementarse en una orientación vertical u horizontal [7].

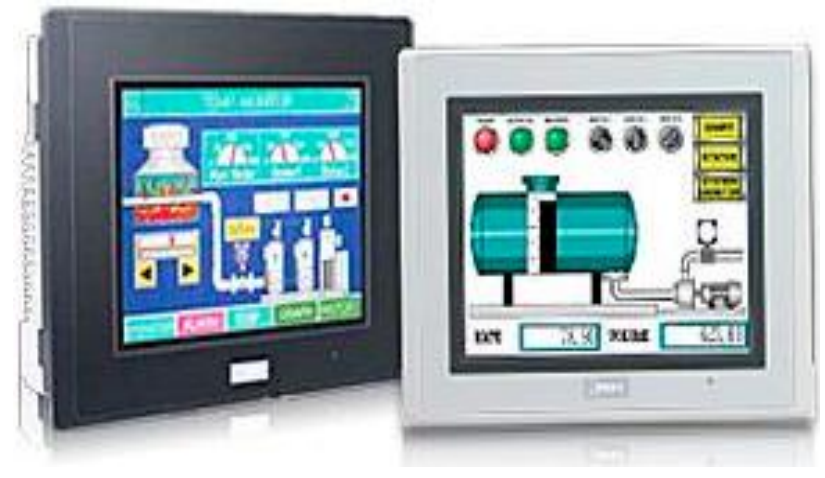

Figura 2 Panel táctil HGIF de IDEC

En la pantalla táctil se podrá modificar parámetros como velocidad de dispensado o velocidad de curado dependiendo de las necesidades del producto. Todos los drivers de los servos motores estan controlados por medio de la recolección de información por equipo de adquisición de datos (RTU), los cuales están controlados por un PLC. Para el PLC se escogió el modelo FC5A-C24R2 de la línea IDEC (ver Fig. 3), que tiene la capacidad de expansión a cuatro módulos, cuenta con 14 entradas y 10 salidas, así como un rango I/O de 88 [9].

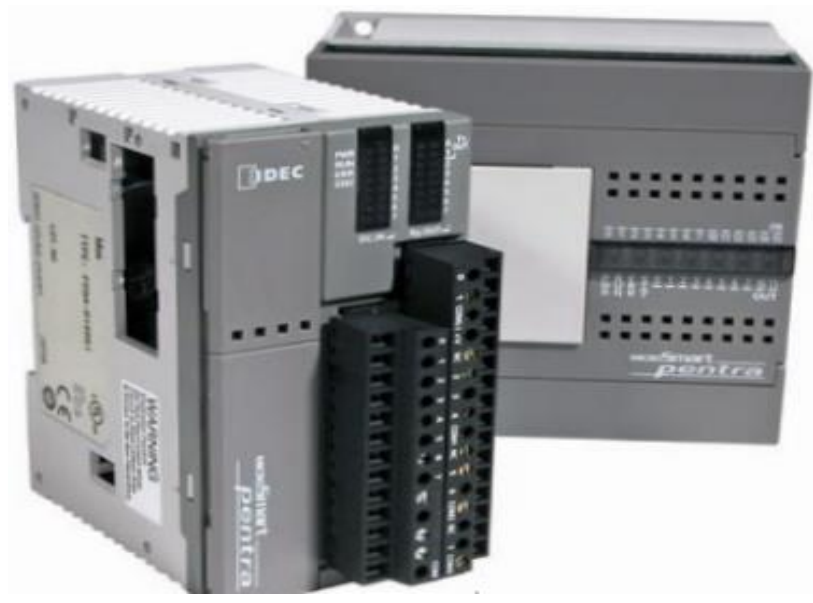

Figura 3 PLC FC5A-C24R2 de IDEC

\section{Resultados}

Solid Works representa una parte esencial dentro del área de diseño, es una herramienta bastante amigable y fácil de utilizar, aunque existen otros software de diseño mecánico como puede ser AUTOCAD y otros no tan conocidos, donde es criterio propio de la industria en particular para usar en este proceso de diseñar fixtures. Fue sencillo implementarla y de este modo obtener una simulación del equipo diseñado y automatizado previniendo así errores que se pueden analizar virtualmente con el fin de evitarlos en el proceso físico. 
Como se mencionó anteriormente, la maquinaria se subdividió en dos estaciones; la estación 1 es el inicio de la operación y es la que se encuentra al lado izquierdo de la mesa, esta cuenta con una unidad de dispensado y 2 etapas de curado del adhesivo. (Ver Fig. 4).

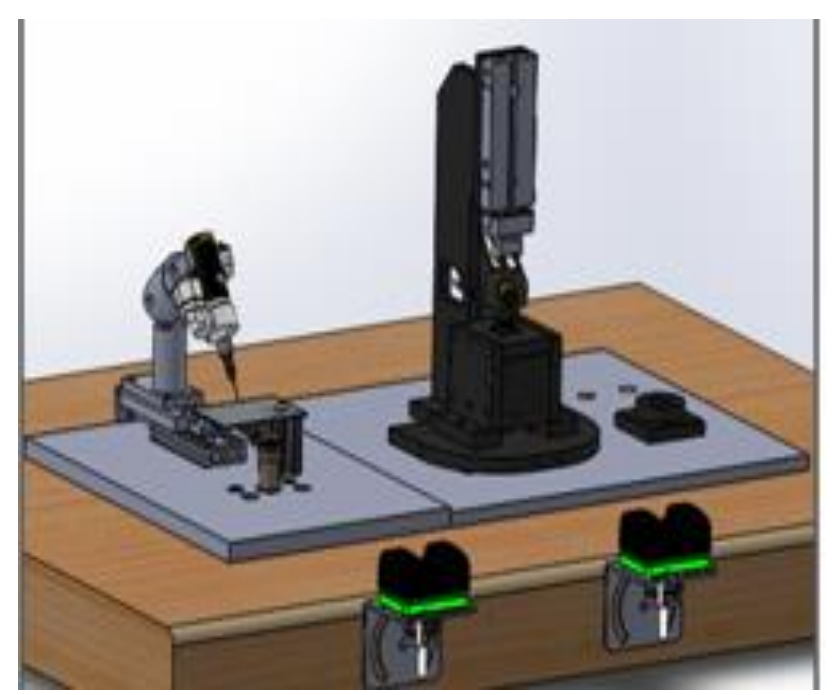

Figura 4 Estación 1

Ya insertado el tubo en fixture se gira la pieza para alinear la aguja con el notch, cuando estos se encuentren alineados se encenderá la luz verde del opty-touch intermitentemente (Ver Fig. 5). Cabe mencionar que el notch se alinea por medio de un sensor óptico, este se encuentra alineado con la punta de la aguja.

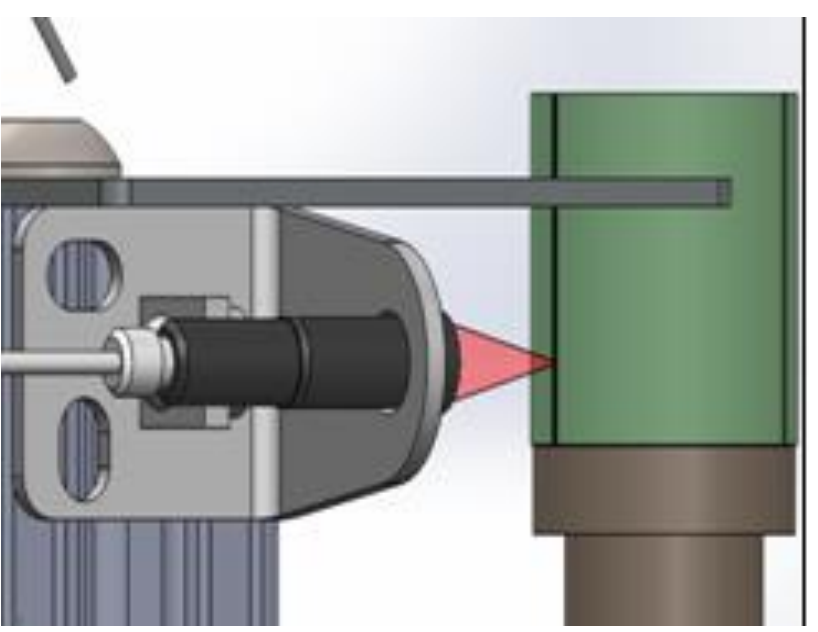

Figura 5 Alineamiento del notch con sensor óptico

En caso de que el sensor presente fallas de detección, se debe ajustar el amplificador. Una vez que el pistón este en posición, se activa el dispensador y girará el fixture con la pieza para agregar adhesivo por todo el contorno del tubo.Ya terminado el proceso de dispensado de adhesivo, se retira la pieza y se coloca en fixture de la prensa de curado.
Una vez cumplido esto se encenderá el indicador opty-touch intermitentemente, indicando que hay una pieza en fixture.

Se activa el opty-touch, para que el vacío en el fixture superior de la prensa comience, se coloca el cap dentro del fixture e inmediatamente el indicador del botón óptico permanecerá encendido con el cap y el tubo en su posición la prensa bajara uniéndolos; una vez que el pistón se encuentra en su posición encenderá la lámpara led y empezara a girar la prensa. (Ver Fig. 6)

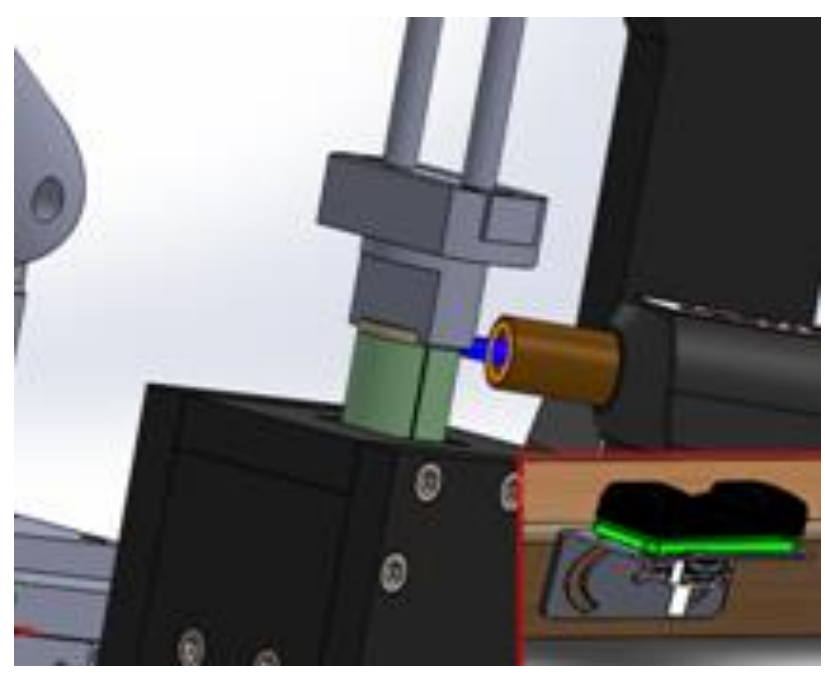

Figura 6 Unión del cap con el tubo por medio de lámparas leds

Una vez terminado el ciclo de curado por el primer extremo de la pieza, se retira del fixture y se inserta en fixture de curado interno, posteriormente se lleva la pieza a la estación 2; dicha estación se encuentra al lado derecho de la mesa, en ella se cura la parte opuesta del tubo y cuenta con un área de dispensado y 2 de curado (Ver Fig.7).

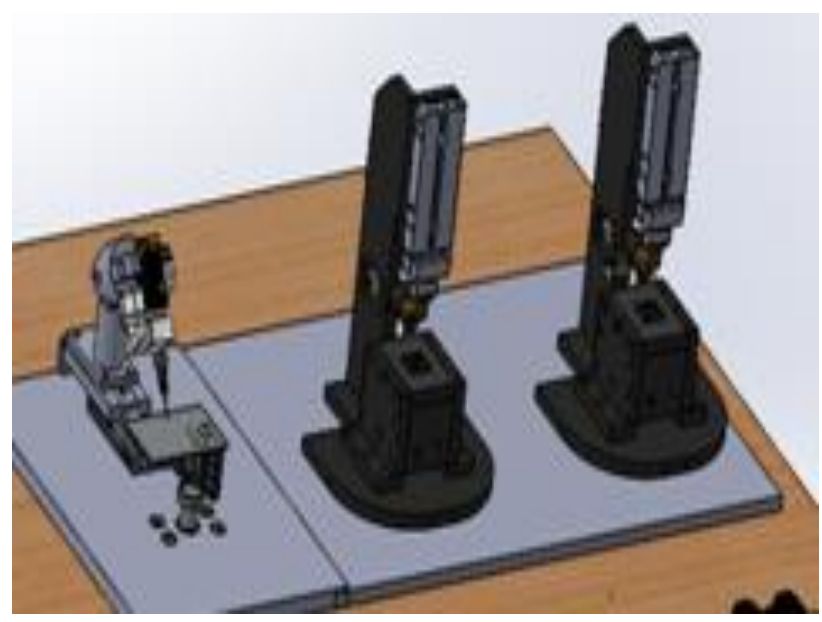

Figura 7 Estación 2 
Se inserta la pieza en el fixture con el extremo ya pegado hacia abajo y se repite la alineación de la aguja con el notch como en la estación 1. Una vez terminada la operación de dispensado se coloca la pieza en fixture inferior de curado de la estación 2 y se repite el proceso de curado como en la estación 1.

Por medio de los CAD fue sencilla la simulación del proceso para poder analizar los resultados que se esperan obtener y es a partir de allí que se llevan a cabo los procesos físicos ya con evidencias, teniendo la certeza de haber cubierto cualquier error que se podría haber presentado.

\section{Conclusiones}

El conjunto de la automatización con el diseño mecánico logra obtener grandes beneficios a la industria. Gracias al diseño, se puede innovar el equipo o maquinaria existente para tener un nuevo resultado en productos finales, reduciendo procesos en uno solo, así como una consistencia con los resultados obtenidos. Con la automatización, la mejora de procesos se logra cuando éstos vienen siendo exigidos en distintos aspectos como lo es el aumento de la productividad, de eficiencia y calidad, así como la reducción de costos y tiempos.

El uso de programas de diseño asistido por computadora (CAD) en el proceso de diseño, es una herramienta de gran beneficio. Gracias a ella, los diseñadores pueden observar resultados sobre el avance del mismo, facilitando la realización de cambios y toma de decisiones respecto a las iteraciones que existen en el proceso de diseño, antes de enviar a manufacturar cualquier pieza. Posterior a la búsqueda de información, análisis y diseño para este sistema, se logró transformar esta celda de dispensado y pegado a un proceso automatizado. Seguidamente de escoger el equipo a utilizar y la metodología, el desarrollo de esquemas y croquis de las piezas a realizar, ayudaron al aumento de calidad y confiabilidad del producto, además de la reducción de la interacción del hombre en el proceso. En la integración y programación se vieron distintos obstáculos como una correcta sincronía e interacción entre los diferentes componentes del sistema, calibración de herramientas, secuencias de tareas y la generación de la lógica.
El desarrollo de este producto no tuvo como base inicial mejorar la productividad de área de trabajo, sino que, como se dijo en la parte de la introducción se realizó para cubrir una necesidad en el proceso de manufactura que fue la de falta de concentricidad que existía entre el cap y su tubo base, por lo que no cubre estudios de tiempos y movimientos para definir como impacta en el proceso productivo.

Estos proyectos pueden ser tomados como referencias por las diversas industrias dedicadas a la manufactura, debido a que cada una presenta desafíos distintos de acuerdo al tipo de producto manufacturado, pero en la mayoría de las veces existen problemáticas comunes que se pueden resolver utilizando la información de diseño de otras industrias y de otros diseñadores.

\section{Referencias}

Aponte Honores, L. M. (2020). Diseño de una microrred fotovoltaica/diésel con backup de batería de Li-Ion para mejorar el servicio eléctrico en la ciudad de San Antonio del Estrecho, Loreto, Perú.

\section{Estévez y I. Garcés, «ESTACIÓN DE VACIADO Y LLENADO,» ESPECTATIVA, 2014.}

Fernández Valencia, S., \& Rodríguez Pérez, K. D. (2020). Diseño preliminar de un sistema par la medición de carga útil en las aeronaves MI17 series.

HENKEL CORPORATION, «LOCTITE,» 2018. [En línea]. Available: https://equipment.loctite.com/product/Dispense -Needle-21-Gauge-1-12-inch-Stainless-SteelHigh-Precision-Purple-50-pk/. [Último acceso: 10 Enero 2018].

IDEC Corporation, «IDEC,» 2018. [En línea]. Available:

http://us.idec.com/Catalog/ProductSeries.aspx? SeriesName $=$ HG1F_Series $\&$ FamilyName $=$ Ope rator_Interfaces. [Último acceso: 10 Enero 2018]. 
IDEC Corporation, «IDEC,» 2018. [En línea]. Available:

http://us.idec.com/Catalog/ProductDetails.aspx

?ProductId=FC5A-

C24R2\&FamilyName $=$ Family $\&$ SeriesName $=$

MicroSmart_Pentra. [Último acceso: 10 Enero 2018].

\section{KEYENCE CORPORATION, «KEYENCE,»}

Enero 2018. [En línea]. Available: https://www.keyence.com.mx/products/sensor/f iber-optic/fs-v20/models/fu-10/index.jsp.

[Último acceso: 10 Enero 2018].

Leal Hortua, A. M. (2020). Propuesta de automatización para bases de datos con macros de excel en el área de fidelización de colmédica medicina prepagada SA.

LOCTITE, «LOCTITE,» Septiembre 2010. [En línea]. Available: http://equipment.loctite.com/documentation/13 90321\%20Integrated\%20Dual\%20Channel\%20 Dispenser\%200-100\%20psi\%20PDS\%20092010.pdf. [Último acceso: 21 Noviembre 2017].

R. Budynas y J. E. Nisbett, DISEÑO EN INGENIERÍA MECÁNICA DE SHIGLEY, Ciudad de México: McGraw-Hill, 2008.

S. Kalpakjian y S. Schmid, MANUFACTURA INGENIERÍA Y TECNOLOGÍA, México: PEARSON EDUCACIÓN, 2008.

Salazar, M., \& Enrique, J. (2020). Propuesta de automatización de la subestación de transmisión Nueva Jaén de Electro Oriente SA. W. E. Deming, Calidad, productividad y competitividad, Madrid: Díaz de Santos, 1989.

Yáñez, J., \& López, C. S. (2020). Innovación en el Equipamiento Didáctico para el Desarrollo de Habilidades y Competencias Profesionales Pertinentes, en el Instituto Tecnológico de Aguascalientes. Pädi Boletín Científico de Ciencias Básicas e Ingenierías del ICBI, 7(14), 78-82. 